\title{
From Spatial to Platial Information Systems: For a Better Representation of the Sense of Place
}

\author{
Yaïves Ferland $^{\mathrm{a},}$ *, Mir Abolfazl Mostafavi ${ }^{\mathrm{b}}$ \\ ${ }^{a}$ Université Laval, Québec, Canada, yaives.ferland@scg.ulaval.ca \\ ${ }^{b}$ Université Laval, Québec, Canada, Mir-Abolfazl.Mostafavi@scg.ulaval.ca \\ * Corresponding author
}

Keywords: Placename, Platial Information System, Toponymic strata, Voronoi hierarchical model, Fuzzy entity limits

\begin{abstract}
:
In a traditional Geographical Information System (GIS), one usually characterizes spatial entities by their individual placename (or toponym) and a set of defined attributes. These entities and their attributes are structured in table frames with functional relations, plus coordinates of their geometric primitives for location in a quantified space. In such a case, one considers the geographical names of these entities as unique identifiers under the standardized 'genericspecific' binomial, in one-to-one relationships with features of interest on the ground. This technical perspective convenes quite well to administrators of land-based public mandates needing univocal references to particular places for planning as well as daily applications (e.g., municipal, postal, emergency). But, from a linguistic point of view, the exact location and dimensions of places are among other attributes of the toponym itself, since the study of placenames looks mainly to its meaning, etymology, and evolution in particular language(s) through the epochs of naming practices, forming kind of toponymic "strata". So, that is investigatory, documented, critical, often anecdotal, sometimes even searching for identity, but devotes few considerations to the geographical place per se, its landscape nor its limits.
\end{abstract}

On the other hand, the geographical perspective to placenames comes more concerned by a real or identifiable place within its vicinity and the dynamics of situations occurring in that location at multiple scales in the same period, whatever the names covering part or all the area. Thus, looking for a place based information system to structure perceived or unofficial places of specified interest require the design and development of tools and functionalities able to support analysis of such information for particular purposes (personal, commercial, military, transit, indigenous, participatory, tourism, etc.). In such a context, definition of their substantial components requires other technical means than their descriptive attributes and geometric primitives. Over the rational spatial databases as a base map, the user of a platial information system would design place-entities on an autonomous fashion, more subjective and colloquial, while referring to landmarks for identity or distinction with respect to different people, being inhabitant, local worker or just visitor. The place-entity comes to the mind like a shapeless mass with a core and some peripheries, where it shows an oriented front or façade, or a force line as a trend, and more fuzzy and moveable limits on the other sides. For instance, downtown and the central business district may not correspond to each other as platial synonyms.

For this purpose, the necessary data structures remain to be developed. To do so, Voronoi tessellation, with its flexible spatial proximity definition and its topological (instead of geometrical) properties, represents an interesting alternative model for further researches. For short, a Voronoi diagram partitions the space into regions such that any location is associated with its nearest Voronoi generators (centroids representing places). This allows an adaptive discretization of the space, and provides a simple and intuitive basis for the definition of adjacency relations between the generation points. Depending on the distribution of generating points, Voronoi cells can approximate place extensions close to human perception of those places with flexibility and still keep the fundamental qualitative relations between places, thanks to topological properties of this model. Irregularity of such Voronoi model also has a significant advantage that allow to better approximate places and their variable distribution in the geographical space.

Here in this paper, we also consider scale as an important factor for the representation and analysis of the places approximated with Voronoi cells. Indeed, in higher level, a place may be constituted either by the aggregation of several places in fine scales or by their parts. Hierarchical Voronoi diagram can also be considered to model such relations between places and their vertical relations, for instance where a same particular placename identifies different features or entities that overlap, evolve, or have various limits or meanings. In such a model, moving between different scales or data "levels" is done without being worried about exact aggregation of lower geometries in a high-level place representation. In order to structure Voronoi hierarchies for a set of points representing centroids of places, one must start by constructing Voronoi cells for the finest level and then creating pointers that relate higher level places to the lower ones. 
Based on its unique properties, Voronoi tessellation, among strong solutions for platial information systems, can provide a firm base for representing the complexity of places, either as entities (or features) and toponyms that identify or refer to them. Paralleling relational GIS data frames, it would allow to adapt to unusual or fuzzy places, to represent their geographical evolution, to preserve their name and spatial extension, and to take good notes of local or ancient variants and even of exonyms applied to such places from abroad. Thus, it presents an opportunity to map the sense, if not the spirit, of place. 